\title{
Hydrologischer Atlas der Schweiz - ein erster Bericht
}

\section{Einleitung}

Die hydrologische Forschung in der Schweiz wird von einem überschaubaren Netz weniger Institutionen und Akteuren geprägt. Da sich die Hydrologie zuerst am realen Gegenstand «Wasser» orientiert und damit institutionelle Strukturen eine sekundäre Rolle spielen, ist die Zusammenarbeit zwischen den Schweizer Hydrologen sehr gut. Sie wird durch eine aktive «Hydrologische Kommission» (SANW) und durch eine initiative «Landeshydrologie und -geologie» weiter gefördert.

Diese Ausgangslage ist für die Realisierung eines umfassenden Projektes, wie es der «Hydrologische Atlas der Schweiz» darstellt, grundlegend wichtig. Damit sind die Voraussetzungen gegeben, das Wasser aus unterschiedlichen Blickwinkeln analysieren zu können (Fig. 1).

Eine Studie von PASQUIER (1977) über die «hydrologische Szene Schweiz» gelangt zum Ergebnis, daß rund $20 \%$ der Aktivitäten im Bereich Niederschlag/ Verdunstung, $30 \%$ im Bereich Boden- und Grundwasser und rund $50 \%$ im Bereich der Oberflächengewässer anzusiedeln sind. Rund ein Drittel davon ist eigentliche Grundlagenforschung. Die restlichen zwei Drittel teilen sich in angewandte (ca. 50\%) und operationelle (ca. 20\%) Hydrologie.

Die Schweiz verfügt im Vergleich mit anderen Staaten über gut ausgebaute, räumlich relativ dichte hydrologische und klimatologische Meßnetze. Die Landeshydrologie und -geologie unterhält rund fünfzig hydrologische Testgebiete.

Die Forschungsförderung durch den Bund, durch die Kantone und vor allem auch durch den Schweizerischen Nationalfonds war und ist beachtenswert. In den letzten Jahren konnte insbesondere das Programm «Grundlegende Probleme des schweizerischen Wasserhaushaltes» realisiert werden. Ein namhafter Kredit steht zur Verfügung, um die Ursachen der Hochwasser 1987 eingehend zu studieren. Ad-hoc-Arbeitsgruppen realisierten bemerkenswerte zusammenfassende Berichte zur Verdunstung (LANG et al. 1978) und zum Niederschlag (SEVRUK et al. 1985). Die Hydrologische Kommission, die Landeshydrologie und -geologie, die Gruppe für operationelle Hydrologie und die zahlreichen Forschungsinstitute sind bemüht, in ihren Mitteilungen

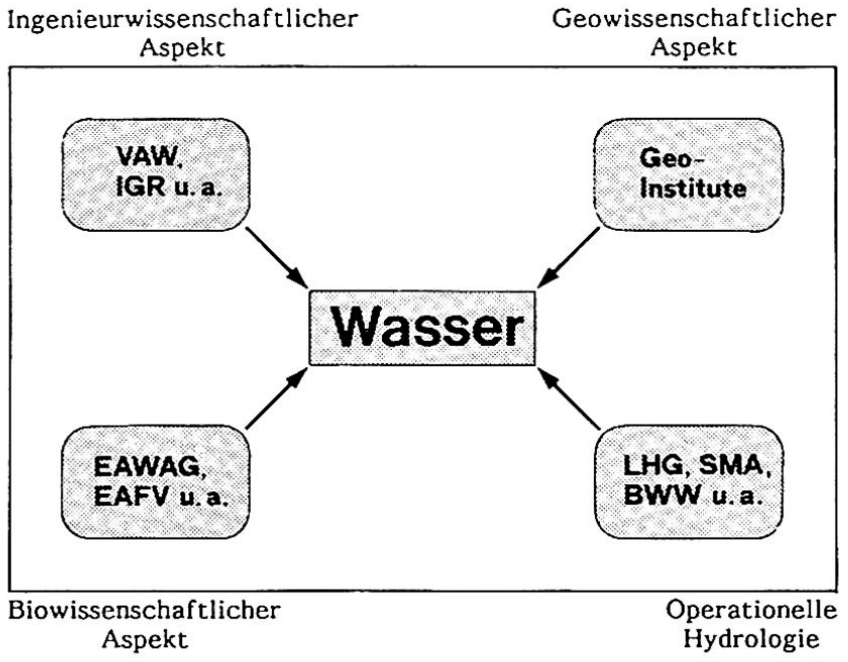

Fig. 1 Die interdisziplinäre Begegnung am Objekt "Wasser» - Situation in der Schweiz

hydrologische Sachverhalte umfassend darzustellen.

Diese notabene nur unvollständige Übersicht läßt erkennen, wie umfangreich und vielfältig die Aktivitäten der schweizerischen Hydrologie sind. Räumlich gesehen reichen die Studien von großmaßstäblichen Analysen kleiner Einzugsgebiete bis zu mittel- bis kleinmaßstäblichen gesamtschweizerischen Untersuchungen.

\section{Zur Bedeutung des «Hydrologischen Atlas der Schweiz»}

Es muß ein Anliegen eines geographisch geschulten und damit primär am Raum orientierten Hydrologen sein, die räumlich breit gestreuten Studien und Informationen zu einem Gesamtbild zusammenzuführen, um damit die großräumigen Zusammenhänge der hydrologischen Phänomene zu erkennen (Fig. 2).

Rolf Weingartner, Dr., Geographisches Institut, Gruppe für Hydrologie, Hallerstr. 12, 3012 Bern 


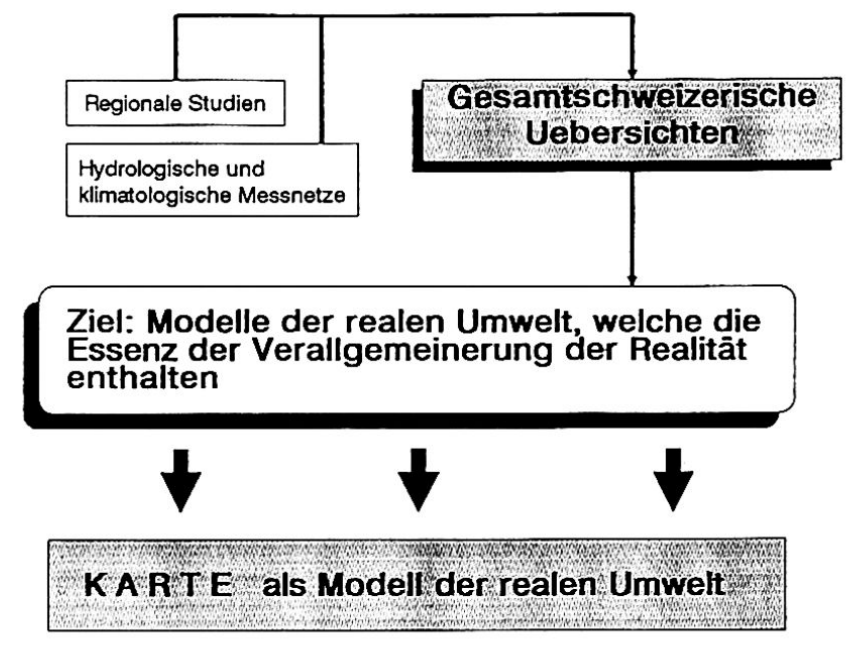

Fig. 2 Die Bedeutung der Karte bei der Umsetzung hydrologischer Sachverhalte

Faszinierend und noch bei weitem nicht ausgeschöpft sind die Möglichkeiten, welche die hydrologischen und hydrologisch relevanten Meßnetze bieten. Damit ist die Frage der Regionalisierung hydrologischer Daten angesprochen, deren Bedeutung heute unbestritten ist. Es sind Ansätze und Methoden gefragt, welche es - besser als bis anhin - ermöglichen, vom Meßpunkt auf die Fläche, von einem gemessenen auf ein ungemessenes Einzugsgebiet zu schließen.

Das Ziel des «Hydrologischen Atlas der Schweiz» ist es also, gesamtschweizerische Übersichten in solchen Betrachtungsmaßstäben zu realisieren, welche es ermöglichen, flächenscharfe Einblicke in hydrologische Phänomene zu gewähren. Damit sind wir auch bei den Fragestellungen der täglichen Praxis angelangt, welche oft in Räumen oder Einzugsgebieten $\mathrm{zu}$ arbeiten hat, die nicht oder nur mangelhaft mit hydrologischen Daten dokumentiert sind. Hier soll und kann der Atlas weiterhelfen. Dazu müssen Modelle der realen Umwelt erarbeitet werden, Modelle, welche die Essenz der Verallgemeinerung der Realität enthalten. Der letzte Teil des vorangehenden Satzes entstammt einer Definition von BOARD (1967), der auf diese Weise thematische Karten definiert. Damit zeigt es sich aber, daß die Karte ein ideales Medium darstellt, um unser Anliegen umzusetzen, nämlich die (Modell-)Ergebnisse einer breiteren Öffentlichkeit zu vermitteln (vgl. Fig. 2). Mit dem «Hydrologischen Atlas der Schweiz» werden somit gesamtschweizerische, flächenscharfe, also räumlich umfassende kartographische Darstellungen wichtiger, wenn immer möglich praxisrelevanter hydrologischer Phänomene angestrebt.

Die Bedeutung von Atlanten als Sammelwerke zusammengehöriger, inhaltlich abgestimmter Kartenblätter (ARNBERGER 1966, IMHOF 1972) hatte bereits

WINKLER (1975) aus der Sicht des leider nicht realisierten «Raumplanungsatlas der Schweiz» hervorgehoben und dabei aufkommende Kritik mit Vehemenz entkräftet: «Man kann ihm [dem «Raumplanungsatlas der Schweiz»] zum Beispiel entgegenhalten, er sei ein zu theoretisches, akademisches Werk, der namentlich den Bedürfnissen des realen, praktischen Planers, der möglichst rasch alle möglichen planlichen Informationen benötige, nicht zu dienen vermöge, zumal er mutmaßlich schneller Veraltung unterliege; er sei vermutlich auch ein in diesem $\mathrm{Zu}$ sammenhang kostspieliges Unternehmen und was der Einwände mehr sind. Ihnen könnte jedoch zweifellos begegnet werden, indem entsprechende organisatorische Maßnahmen zum Beispiel dauernder Nachführung ... getroffen werden. Doch abgesehen davon dürften die positiven Werte eines solchen Werkes die negativen durchaus überwiegen: abgesehen davon, daß es - was sicher nicht unterschätzt werden darf - erlaubt, die Gesamtheit der Planungsinhalte in sachlicher, räumlicher, zeitlicher

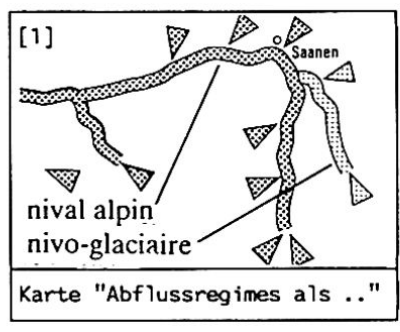

[3]

$$
\begin{aligned}
& A= 1.39^{* N}(S O)-158.45^{*} \mathrm{~N}(\mathrm{SO}-\mathrm{HI})^{2} \\
&-111^{\prime} 142.54^{*} \ln (\mathrm{T}+273.15) \\
&+66.59 * \ln (\mathrm{mV}+1)+62^{\prime} 792 \\
& \text { Formel für die Alpennordseite }
\end{aligned}
$$

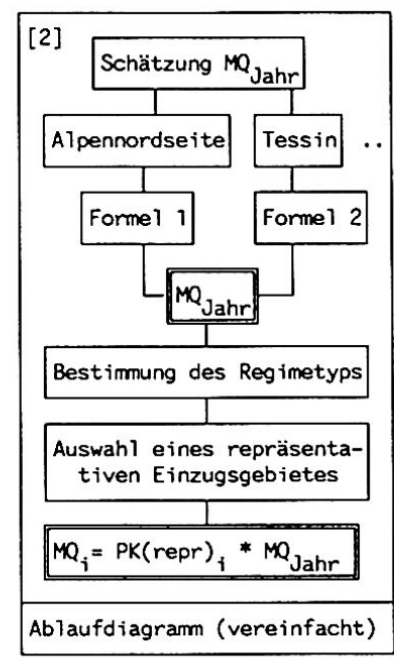

[4]

XARTE "Abflussregimes.." $\longrightarrow$ Saane mit nivalem Regime $\longrightarrow$ repräsentative Einzugsgebiete: Allenbach, Filderich, Muota, Grosstalbach, Plessur $\longrightarrow$ räumliche Analogie und Nachbarschaftsbeziehungen: Wahl des Filderichs als repräsentatives Einzugsgebiet $\longrightarrow$ Übertragung der Pardé-Koeffizienten des Filderichs $($ PK(repr)) auf die Saane

Regimetyp und repräsentatives Einzugsgebiet

$[5]$

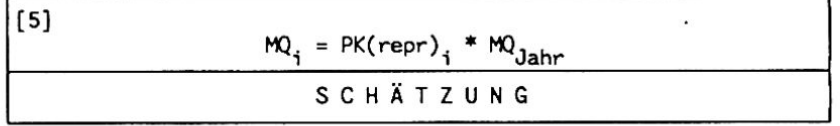

Fig. 3 Zur Abschätzung von langjährigen mittleren Monatsabflüssen mit Hilfe der Tafel "Abflußregimes" des "Hydrologischen Atlas der Schweiz" - Beispiel: Saane in Saanen; Abkürzungen: $N_{(S O)}$ : Gebietsniederschlag im Sommerhalbjahr [mm]; $N_{(S O-w)}$ : Verhältnis Sommer- zu Wintergebietsniederschlag; $\mathrm{T}$ : mittlere Jahrestemperatur $\left[{ }^{\circ} \mathrm{C}\right]$; $\mathrm{mV}$ : mittlere areale Vergletscherung [\%] 
und nicht zuletzt methodisch-systematischer Hinsicht zu überblicken, erlaubt er, wie keine andere Darstellungsweise, Vergleiche, die den Atlas allein schon unentbehrlich erscheinen lassen.»

Schon mehrmals wurde die Praxis angesprochen. Im folgenden Beispiel soll deshalb eine Möglichkeit aufgezeigt werden, wie mit dem «Hydrologischen Atlas der Schweiz» beispielsweise gearbeitet werden kann:

Fragestellung: Es sollen für die Saane in Saanen die langjährigen mittleren Monatsabflüsse in der $\mathrm{Di}$ mension $\left[\mathrm{m}^{3} \mathrm{~s}^{-1}\right]$ abgeschätzt werden.

Vorgehen (s. Fig. 3): Ausgangspunkt bildet die Atlastafel «Abflußregimes als Grundlage zur Abschätzung von Mittelwerten des Abflusses», welche als Pilotkartenblatt des «Hydrologischen Atlas der Schweiz» bereits vorliegt (ASCHWANDEN und WEINGARTNER 1985). Das «Layout» des Atlasses sieht neben der eigentlichen Karte im Maßstab 1:500 000 auch einen Erläuterungstext im Umfang von drei A4-Seiten sowie Graphiken und Tabellen vor.

Auf der Graphik- und Tabellenseite der Tafel findet der Anwender nun ein Ablaufdiagramm, das ihm aufzeigt, wie er vorzugehen hat (Fig. 3:2):

Als erstes muß er den langjährigen mittleren Jahresabfluß abschätzen (nach ASCHWANDEN 1985). Die Karte und das Ablaufdiagramm liefern die Grundlagen zur Wahl der geeigneten Abschätzformel. Für die Saane in Saanen ist die für die Alpennordseite gültige Schätzformel zu wählen, die ebenfalls auf der Graphik- und Tabellenseite zu finden ist (Fig. 3: 3).

In einem nächsten Schritt (Fig. 3: 4) muß der Anwender mit Hilfe der Karte den Regimetyp, welche die Saane in Saanen aufweist, feststellen: Es handelt sich um ein alpin nivales Regime (Fig. 3: 1). Wie alle andern fünfzehn Regimetypen der Schweiz wird auch das nivale Regime durch mehrere repräsentative Einzugsgebiete beschrieben. Diese sind auf der Tafel zusammen mit wichtigen Einzugsgebietsparametern und mit den mittleren Pardé-Koeffizienten ausgewiesen. Aufgrund räumlicher Analogien und Nachbarschaftsbeziehungen wählen wir aus diesen den Filderich in Riedli aus. Vom Filderich, der in der Folge als repräsentativ für die Saane angesehen wird, werden dann die Pardé-Koeffizienten (PK) übernommen bzw. übertragen. Die PardéKoeffizienten sind definiert als:

$\mathrm{PK}_{\mathrm{i}}=\frac{\mathrm{MQ}_{\mathrm{i}}}{\mathrm{MQ}_{\mathrm{JAHR}}}[-]$

mit $\mathrm{PK}_{\mathrm{i}}: \quad$ dimensionsloser Pardé-Koeffizient $\mathrm{MQ}_{\mathrm{i}}: \quad$ langjähriger mittlerer $A b f l u ß$ des i-ten Monats

$\mathrm{MQ}_{\mathrm{JAHR}}$ : langjähriger mittlerer Jahresabfluß
Anschließend multiplizieren wir den geschätzten Jahresabfluß mit den übertragenen Pardé-Koeffizienten (Fig. 3: 5) und erhalten als Ergebnis eine Abschätzung der langjährigen mittleren Monatsabflüsse. Wie die Fig. 4 abschließend zeigt, gelangt man mit diesem Ansatz in der Regel zu guten Ergebnissen.

\section{Saisonale Abflussverteilung} Saane-Saanen

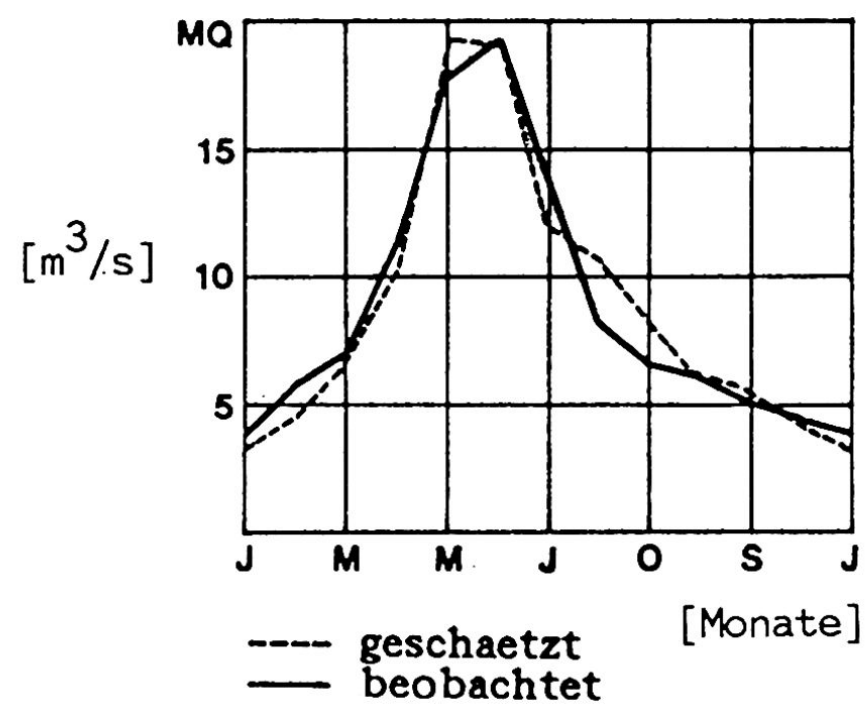

Fig. 4 Vergleich zwischen den geschätzten und den tatsächlich beobachteten langjährigen Monatsmitteln des Abflusses der Saane in Saanen

Insgesamt konnte die Abschätzung der Monatsmittel der Saane dank der Gestaltung der Atlas-Tafel, welche eine Integration zusätzlicher wichtiger und nützlicher Informationen ermöglicht, weitgehend autonom erfolgen.

In Zusammenfassung und Erweiterung des Gesagten läßt sich die Bedeutung des «Hydrologischen Atlas der Schweiz» wie folgt umschreiben:

auf der Seite der Forschung:

- neue Forschungsimpulse z. B. im Bereich der Regionalisierung;

- Standortbestimmung der Hydrologie, u.a. Erkennen von Kenntnis- und damit auch Forschungslücken;

- weitere Förderung der Zusammenarbeit zwischen den Hydrologen.

auf der Seite der Praxis.

- Erarbeitung von flächendeckenden, praxis- und planungsrelevanten Ergebnissen;

- Erleichterung des Datenzugriffs: Karte als Datenspeicher, Bereitstellung von Karten über die hydrologischen Meßnetze;

- Erleichterung des Einstiegs in regionale Untersuchungen: grundlegende Zusammenhänge - wie 
beispielsweise die Größenordnung des zu erwartenden Gebietsabflusses - werden im Atlas aufgezeigt.

\section{allgemein:}

- Sensibilisierung einer breiteren Öffentlichkeit für hydrologische Fragestellungen.

\section{Konzeptionelles}

Der «Hydrologische Atlas der Schweiz» ist eine systematisch abgestimmte Folge hydrologischer Kartenblätter. Die Systematik liefert uns die Gliederung der Hydrologie nach ihren Systemelementen. Diese bilden die Grundeinheiten des Atlasses. Sie weisen einen quantitativen (Menge) und einen qualitativen (Beschaffenheit) Aspekt auf. Damit ergibt sich folgendes Inhaltsverzeichnis:
1 Grundlagenkarten
5 Abfluß und Seen
2 Niederschlag
6 Wasserhaushalt
3 Schnee und Gletscher
4 Verdunstung
7 Stoffhaushalt
8 Boden- und Grund- wasser

Diese Anordnung der Karten erlaubt eine größtmögliche Flexibilität. Die Kartenblätter werden dezimal beschriftet (z. B. 2.1 Niederschlagsmeßnetz) und in einem Ringordner abgelegt. Neu aufgelegte oder später gelieferte Kartenblätter lassen sich somit problemlos an der entsprechenden Stelle einordnen. Die Einheit des Atlas bleibt stets erhalten. WINKLER (1953) hat die Begriffe konstitutive und dispositionelle Karten geprägt. Konstitutive Karten beschreiben die «Beschaffenheit eines Faktors an sich», während dispositionelle Karten unmittelbar das Potential eines Raumes aufzeigen. Letztere dürfen nach WINKLER «keinesfalls als Inventarkarten üblichen Sinns, sondern als Eignungs- und Risikokarten konzipiert werden». Aus hydrologischer Sicht haben beispielsweise folgende Themen dispositionellen Charakter: Eignung der Gewässer für gesellschaftliche Ansprüche; Risiken, welche von Gewässern ausgehen: Überschwemmungen, Wassermangel, Verschmutzung usf.; Schutzwürdigkeit und Schutzbedarf der Gewässer u. a.m.

Es versteht sich, daß in der ersten Bearbeitungsphase des Atlasses (1989-1991) vor allem Kartenblätter konstitutiven Inhaltes realisiert werden können. In einer Vorstudie und in Gesprächen wurden für die erste Atlas-Phase folgende Kartenblätter festgesetzt:

\subsection{Topographische Übersicht}

1.2 Übersicht über geologische, hydrogeologische und pedologische Karten

1.3 Hydrologische Gliederung der Schweiz

\subsection{Niederschlagsmeßnetze}

2.2 Systematischer Niederschlagsmeßfehler 1951-1980

2.3 Mittlere jährliche Niederschlagshöhen 1951-1980

2.4 Höhe, Dauer und Wiederkehrperiode extremer Punktregen

3.1 Schnee- und Gletschermeßstationen

3.2 Schnee: Betrachtung extremer Zustände

3.3 Wasseräquivalent der Schneedecke

4.1 Verdunstungsmeßnetze und potentielle Verdunstung

5.1 Hydrometrische Stationen

5.2 Abflußregimes - Grundlage zur Abschätzung von Mittelwerten des Abflusses

5.3 Beeinflussung der Fließgewässer durch Kraftwerke und Seeregulierungen

6.1 Wasserbilanzen großer Einzugsgebiete 1901-1980

6.2 Gebietswasserhaushaltliche Darstellungen $1961-1980$

7.1 Meßpunkte physikalischer und chemischer Parameter

7.2 Physikalische und chemische Parameter an Oberflächengewässern

Diese Prioritäten in der Bearbeitung ergaben sich aufgrund des Status einer Karte (konstitutiv oder dispositionell), aufgrund der Datenlage (Daten vorhanden?) und aufgrund des Arbeitsaufwandes (Arbeitsaufwand $<3$ Jahre).

Alle raumbezogenen Daten lassen sich mit den drei Dimensionen Phänomen (Merkmalsart), Raum (Lage) und Zeit (Zeitpunkt) erfassen. BERRY (1964) hat diese geographische Totalität mit dem sogenannten Datenwürfel beschrieben (Fig. 5). Aus dem Datenwürfel lassen sich zweidimensionale Matrizen extrahieren. Für die kartographische Darstellung sind dabei die räumlich-sachliche Matrix - unter Konstanthaltung des Zeitpunktes oder des Zeitraumes - und die historisch-genetische Matrix - unter Konstanthaltung des Merkmals - relevant.

Die räumlich-sachlichen Darstellungen werden den Atlasinhalt dominieren. Es wird hier aufgezeigt, wie ein Merkmal während einer bestimmten Zeitperiode im Raum verteilt ist. Als Beispiel kann die Tafel «2.3 Mittlere jährliche Niederschlagshöhen 1951-1980» genannt werden. Als Standardperiode wurde für den «Hydrologischen Atlas der Schweiz» der Zeitraum 1951-1980 gewählt, wobei nicht absolut starr daran festgehalten werden soll. Methodisch können räumlich-sachliche Inhalte, an die der Anspruch einer räumlich umfassenden Darstellung gestellt wird, mittels regional-taxonomischer Ansätze oder nach dem Prinzip der regionalen Übertragungsfunktionen erarbeitet werden. Die Fig. 6 vermittelt dazu weitere Anhaltspunkte. 
Mit der LT-Matrix wird die räumlich-zeitliche Entwicklung eines Phänomens beobachtet; dies kann retrospektiv oder prognostisch geschehen. Prognosen haben bereits dispositionellen Charakter.

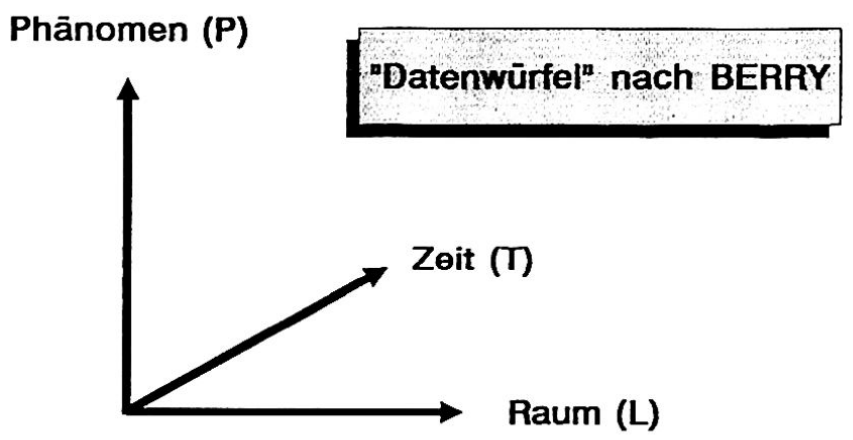

\section{2-dimensionale Matrizen}

T=konst.

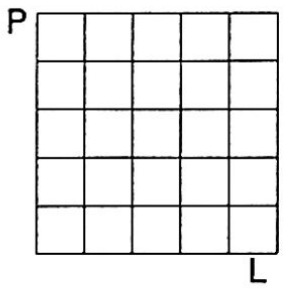

RÄUMLICH-SACHLICH

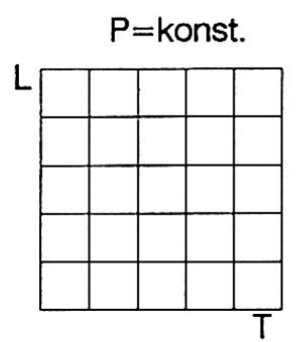

HISTORISCH-GENETISCH; PROGNOSTISCH

Fig. 5 Der Datenwürfel nach BERRY (1964) und daraus abgeleitete, kartographisch umsetzbare zweidimensionale Matrizen

Bei der Realisierung einer Karte bzw. eines Atlaswerkes ist natürlich die Frage des Kartenmaßstabs grundlegend wichtig. Der Maßstab entscheidet letztlich über die Möglichkeiten und Grenzen der Aussagen.

Als Hauptmaßstab des «Hydrologischen Atlas der Schweiz» wurde $1: 500000$ gewählt. Er drängt sich aus verschiedenen Gründen auf:

- Er erlaubt es einmal, hydrologische Aussagen in einer noch praxistauglichen Dimension zur Darstellung zu bringen. Fig. 7 hält die Zusammenhänge zwischen Fläche und Maßstab einerseits und den hydrologischen Aussagemöglichkeiten andererseits fest, Zusammenhänge notabene, wie sie in einer Studie der UNESCO-WMO (1977) aufgedeckt wurden. Nach einer Zusammenstellung von SANDNER und BIELER (1981) sind in diesem Hauptmaßstab Mikro- und Mesochoren noch darstellbare Kartierungseinheiten. Nach denselben Autoren können Karten dieses Maßstabes zur "planerischen Landschaftsdiagnose auf nationaler und regionaler Ebene» verwendet werden. Diagnostisch wird hier synonym $\mathrm{zu}$ dem oben besprochenen Begriff «dispositionell» verwendet.

- Er ist mit dem Hauptmaßstab des «Atlas der Schweiz» identisch und erlaubt damit einen einfachen Daten- und Informationstransfer. Die beiden Atlanten wie auch weitere Atlanten - so der «Klimaatlas der Schweiz» - sollen sich nicht konkurrenzieren, sondern vor allem ergänzen.

- Er ermöglicht ein Kartenformat, das dem Atlas noch eine gewisse Handlichkeit gibt.

Der Hauptmaßstab 1:500000 muß aus wissenschaftlicher Sicht als nicht zu unterschätzende Her-

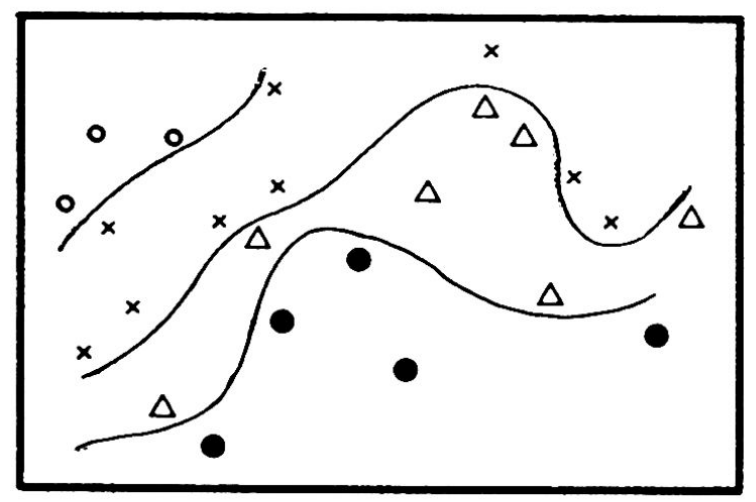

"Regionale Taxonomie"

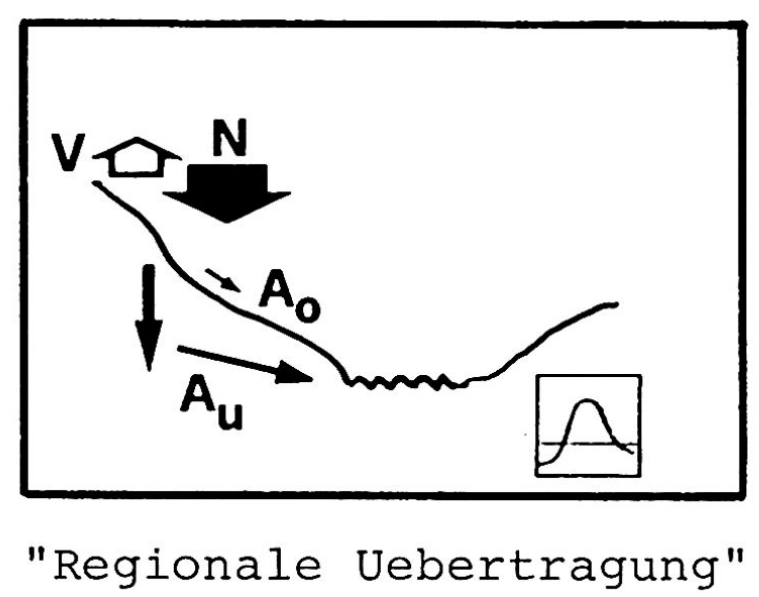

Fig. 6 Regionale Taxonomie: “. . . Typisierung von Raumeinheiten. Durch die Typisierung werden in der Regel Raumeinheiten zu Gruppen nach Maßgabe einer Ähnlichkeitsdefinition zusammengefaßt."

Regionale Übertragung: “. . . die Anwendung hydrologischer Modelle auf Gebiete, für die sie mangels geeigneter Meßdaten nicht direkt kalibriert, sondern nur indirekt unter Berücksichtigung gebietsspezifischer Charakteristika angepaßt werden können." (SCHWENTKER und STREIT 1983) 

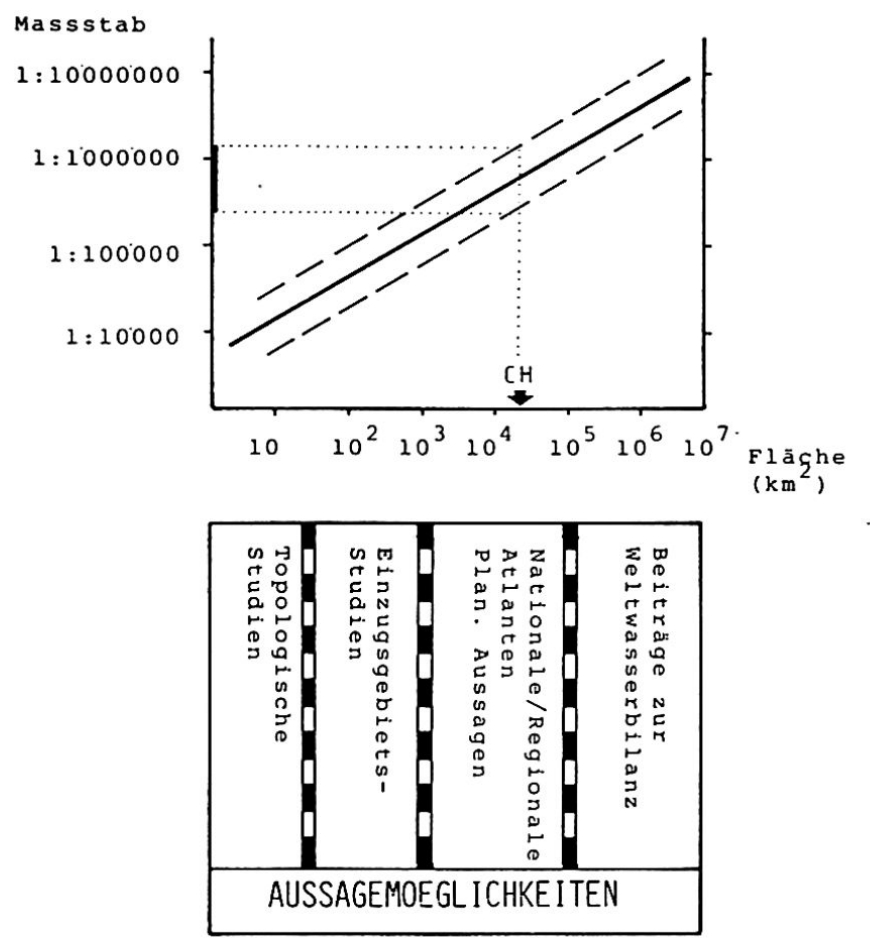

Fig. 7 Der Zusammenhang zwischen der Fläche eines Landes und dem optimalen Kartenmaßstab im Vergleich mit den hydrologischen Aussagemöglichkeiten (n. UNESCO-WMO 1977, modifiziert)

ausforderung angesehen werden; er verlangt nämlich räumlich relativ hochauflösende Informationen, die im Alpenland Schweiz oft nur schwer bereitzustellen sind. Auf der andern Seite ist eine Generalisierung, wie sie ein Maßstab 1:500 000 erfordert, notwendig und ehrlich, da sich beispielsweise hydrolgische Phänomene von Hang zu Hang verändern können; diese Veränderungen lassen sich aber nach dem heutigen Erkenntnisstand in den wenigsten Fällen über große Räume hinweg detailliert wiedergeben.

In jenen Fällen, in denen das Wissen oder die Daten den Hauptmaßstab nicht verantworten lassen, kann auf kleinere Maßstäbe ausgewichen werden: $1: 800$ 000, 1:1,1 Mio., 1:1,4 Mio., 1:1,7 Mio., $1: 2,2$ Mio., $1: 3$ Mio.

\section{Rückblick und Aúsblick}

Die ersten Konzepte für den «Hydrologischen Atlas der Schweiz» wurden in den frühen 1980er Jahren erarbeitet (WEINGARTNER 1985). Es zeigte sich dabei, daß die hydrologische Kartographie in der Schweiz - vor allem was gesamtschweizerische Karten betrifft - meist in den Anfängen steckengeblieben ist. Dies erstaunt um so mehr, als die Karte das Medium darstellt, um (hydrologische) Forschungs- ergebnisse schnell und prägnant einer breiteren Öffentlichkeit zugänglich zu machen. Einzig auf dem Gebiet des Niederschlags kann man bereits von einer gewissen Tradition sprechen (WEINGARTNER 1989). Mit dem «Hydrologischen Atlas der Schweiz» sollen diese Defizite gedeckt und ein Medium bereitgestellt werden, das sowohl der Lehre und Forschung als auch der Praxis und einer breiteren Öffentlichkeit dienen kann.

Der Atlas wird als Gemeinschaftswerk der Schweizer Hydrologen realisiert. Als Autoren konnten die in der jeweiligen Thematik beheimateten Fachspezialisten gewonnen werden. Es handelt sich somit beim Atlas auch um einen Leistungsausweis der schweizerischen Hydrologie.

Die Arbeiten zum Atlas wurden auf Jahresbeginn 1989 aufgenommen. Die Projektleitung liegt in den Händen der Gruppe für Hydrologie des Geographischen Institutes der Universität Bern. Die AtlasKommission, welche sich aus den Vorstehern der am Atlas beteiligten Institutionen zusammensetzt, ist für die Behandlung technischer und finanzieller Angelegenheiten zuständig. Finanziert wird das Projekt durch die Landeshydrologie und -geologie. Das Patronat liegt in den Händen der Hydrologischen Kommission der SANW und der Gruppe für operationelle Hydrologie. Für den Kartendruck konnte das Bundesamt für Topographie $(\mathrm{L}+\mathrm{T})$ gewonnen werden. Für den Vertrieb des Atlas ist die Eidg. Druck- und Materialzentrale (EDMZ) zuständig.

Die erste Lieferung des Atlasses, welche rund zwanzig Tafeln umfassen wird, ist 1992 zu erwarten. Die Kartenblätter werden nicht einzeln verkauft und auch nicht im voraus veröffentlicht. Der Verkaufspreis dürfte sich bei Fr. 200.- bewegen.

Gegenwärtig ist die Planung der zweiten Phase im Gange, in der vermehrt auch dispositionelle Sachverhalte angegangen werden sollen.

Unsere Kontakte im ersten Bearbeitungsjahr haben gezeigt, daß die Nachfrage nach gesamtschweizerischen, räumlich detaillierten hydrologischen Informationen groß ist. Erfreulich ist der "Goodwill», der dem Projekt entgegengebracht wird. Es bleibt zu hoffen, daß die Projektarbeiten planmäßig ablaufen und daß der «Hydrologische Atlas der Schweiz» ab 1992 in regem Gebrauch sein wird.

\section{Literatur}

ARNBERGER, E. (1966): Handbuch der thematischen Kartographie, Wien.

ASCHWANDEN, H. (1985): Zur Abschätzung der Abflüsse in ungemessenen schweizerischen Einzugsgebieten. Publikationen Gewässerkunde Nr. 66, Bern.

ASCHWANDEN, H., WEINGARTNER, R. (1985): Die Abflußregimes der Schweiz. Publikationen Gewässerkunde Nr. 65, Bern. 
BERRY, B. (1964): Approaches to Regional Analysis: a Synthesis. Annals Association of American Geographers 54: 2-11.

BOARD (1967): zitiert in BRASSEL, K.: Kurs in Karteninterpretation, Zürich.

IMHOF, E. (1972): Thematische Kartographie. Lehrbuch der Allgemeinen Geographie, Band X, Berlin.

LANG, H., et al. (1978): Die Verdunstung in der Schweiz. Beiträge zur Geologie der Schweiz - Hydrologie, Nr. 25, Bern.

PASQUIER, F. (1977): Rapport sur la recherche hydrologique en Suisse, Neuenburg.

SANDNER, E., BIELER, J. (1981): Zur Konzeption geoökologischer Karten für die Landschaftsdiagnose und Landschaftsplanung. In: Petermanns Geographische Mitteilungen 1981/1: 63-68, Gotha.

SCHWENTKER, F., STREIT, U. (1983): Regionale Übertragung hydrologischer Parameter - ein Überblick. Vortragsmanuskript Arbeitskreis Hydrologie, Göttingen.
SEVRUK, B., et al. (1985): Der Niederschlag in der Schweiz. Beiträge zur Geologie der Schweiz - Hydrologie, Nr. 31, Bern.

UNESCO-WMO (1977): Hydrological Maps - a Contribution to the International Hydrological Decade. Studies and Reports in Hydrology Nr. 20, Paris.

WEINGARTNER, R. (1985): Konzept eines «Hydrologischen Atlas der Schweiz". Publikationen Gewässerkunde Nr. 67, Bern.

WEINGARTNER, R. (1989): Precipitation Maps in the "Hydrological Atlas of Switzerland" - Historical Survey and Actual Significance. International Workshop of Precipitation Measurement (IAHS, WMO, ETH): 527-530, St. Moritz.

WINKLER, E. (1953): Regionalbeschreibung der Schweiz als Grundlage geographischer Landesaufnahme und Landesplanung. In: Geographica Helvetica VIII: 266-269, Zürich.

WINKLER, E. (1975): Der Raumplanungsatlas der Schweiz. Vermessung, Photogrammetrie, Kulturtechnik 1975: 1-75, Zürich. 